\title{
Quand Rancière s'invite chez le médecin : expertise, ignorance et émancipation dans un dispositif participatif de soin
}

\author{
Alexandre Fauquette
}

\begin{abstract}
Résumé
L'article s'intéresse à un dispositif d'éducation thérapeutique du patient conçu et imaginé par un médecin libertaire qui se réfère au cadre pédagogique du maître ignorant de Jacques Rancière pour rompre radicalement avec les codes traditionnels de la relation médicale et effacer toute forme d'imposition d'un biopouvoir. Il analyse plus spécifiquement les ambiguïtés qui en découlent : si l'espace de participation de ce dispositif de soin alternatif tend à réduire l'asymétrie de la relation médicale, il n'émancipe cependant que les émancipés et ne parvient à rompre totalement avec les formes de domination médecin-patient. L'égalitarisme ranciérien est en effet mis à l'épreuve de la pratique et de la responsabilité médicale, et nécessite des compromis incessants avec l'institution hospitalière. Mais les marges de manœuvre laissées par l'institution hospitalière renforcent dans le même temps le caractère transgressif et buissonnier de ce dispositif.
\end{abstract}

«Comment renforcer la participation de nos patients dans nos consultations médicales ?» Telle est la question que se sont posée, au cours de l'une de leurs réunions de service hebdomadaires, les professionnels de santé d'un centre de nutrition en milieu hospitalier, le Cétrabète (un centre d'éducation et de traitement des maladies liées à la nutrition et au diabète) ${ }^{1}$. Cette conception individualisée ${ }^{2}$ de la participation renvoie à des pratiques particulières du soin qui tendent à se développer de manière exponentielle du fait, entre autres, de la chronicisation rampante d'un certain nombre de pathologies qui réduit le pouvoir de guérison des professionnels de santé, rendent la communication entre patients et professionnels de santé plus fréquente (Van Ballekom, 2008), et obligent certains patients à participer activement et quotidiennement à leur traitement ${ }^{3}$. On trouve en effet dans le secteur médical des expériences de participation où des patients et des professionnels de santé expérimentent d'autres formes de consultation. C'est notamment le cas dans le service de nutrition du docteur Millot dans un hôpital de l'agglomération lilloise. Chaque année, ce jeune praticien hospitalier met en place, avec son équipe pluridisciplinaire de soins ${ }^{4}$, des dispositifs

\footnotetext{
${ }^{1}$ Le nom du service et les noms de famille des personnes ont tous été modifiés pour respecter leur anonymat.

${ }^{2}$ Cette conception individualisée de la participation en santé se distingue ici d'une conception plus collective et politique de la participation, telle qu'on peut la trouver, par exemple, dans l'activisme thérapeutique des patients atteints du VIH dans les années 1980, ou encore dans les pratiques communautaires en santé.

${ }^{3}$ Nous pouvons bien évidemment avancer d'autres facteurs explicatifs. L'activisme thérapeutique des malades du sida (Barbot, 2002) a par exemple concouru à faire progresser les droits des patients. On peut aussi noter l'émergence d'un discours crisologique qui, depuis le début des années 1980, s'attaque à la déshumanisation de la médecine (Azria, 2013) et propose de repenser la relation médicale sur un mode plus horizontal.

${ }^{4}$ Composée de deux médecins nutritionnistes, d'un diététicien, d'une psychologue, d'un éducateur médicosportif et d'une infirmière d'éducation.
} 
«d'éducation thérapeutique du patient » (que nous nommerons sous le sigle $\mathrm{ETP}^{5}$ ) ainsi que certains groupes de parole, en particulier un groupe de thérapie comportementale sur les troubles des conduites alimentaires, qui cassent les codes traditionnels de la relation médicale : conformément au «maître ignorant » de Jacques Rancière (2004), le professionnel de santé ne cherche pas à imposer ou à enseigner son savoir médical à ses patients, mais à faire en sorte, comme l'affirme le docteur Millot lui-même, «d'accompagner le patient pour que celui-ci trouve en lui-même les ressorts de son mieux-être ».

\section{Encadré 1. Le cadre pédagogique de la pensée de Jacques Rancière dans Le maître ignorant}

Dans son ouvrage Le maître ignorant (1984), Jacques Rancière développe une pensée philosophique originale sur l'éducation et le politique. Il tire cette pensée de l'expérience éducative vécue par Joseph Jacotot, un professeur qui fit scandale dans la France et la Hollande du milieu du XIX ${ }^{\mathrm{e}}$ siècle après avoir proclamé que les élèves pouvaient apprendre seuls, c'est-à-dire sans maître pour leur expliquer, et que, de leur côté, les maîtres pouvaient enseigner ce qu'ils ignoraient eux-mêmes. Cette proclamation lui vint de son expérience d'enseignement au cours de laquelle il dut enseigner à des élèves flamands dont il ne connaissait pas la langue, ni eux la sienne, un ouvrage bilingue. Après leur avoir demandé de lire l'ouvrage en s'aidant de la traduction bilingue, il leur enjoint d'écrire en français ce qu'ils en pensaient et fut surpris de constater ce qu'ils avaient appris par eux-mêmes, et de ce qu'ils étaient eux-mêmes capables de lui apprendre. D'où l'idée que les élèves peuvent se passer d'un maître et que celui-ci est, au mieux, un « maitre ignorant » qui enseigne sans transmettre aucun savoir, si ce n'est éventuellement un savoir qu'il ignore. Quelle conclusion en tire Rancière ? Qu'il faut rompre avec le recours habituel à l'explication dans la pratique éducative. La tentation de vouloir expliquer à autrui porte en effet en elle le germe de l'inégalité puisqu'elle divise fondamentalement le monde en deux : les sachants d'un côté et les ignorants de l'autre, ceux qui expliquent et ceux qui écoutent, les premiers éclairant les seconds d'un savoir qu'ils estiment être seuls à posséder. Or, l'expérience de Jacotot démontre qu'il n'y a pas une inégalité des savoirs et des intelligences, mais au contraire une égalité des intelligences qui doit être mise en pratique dès le départ, "même sans preuves » (Cerletti, 2005). En ce sens, et c'est là toute la portée politique de sa pensée, l'égalité ne doit pas être une fin en soi mais un point de départ. Ce qui rompt radicalement avec toute action progressiste qui vise à faire de la réduction des inégalités une fin en soi, à défaut de faire de l'égalité le point de départ des possibles.

Même si, à la différence du maitre ignorant de Rancière, le médecin dispose d'un savoir expert et qu'il n'est pas, à l'instar de Joseph Jacotot, un maître qui enseigne ce qu'il ignore, il s'inscrit tout de même en porte-à-faux à l'égard de la verticalité de l'explication en ce qu'elle est un premier axiome d'inégalité. Il proclame ainsi l'horizontalité des rapports sociaux. Il s'agit en effet de modifier les codes traditionnels de la relation médicale, d'effacer la frontière entre le sachant et l'ignorant grâce à des dispositifs qui se veulent horizontaux et « réellement émancipateurs ». C'est à ces dispositifs particuliers d'éducation thérapeutique du patient mis en place dans ce service hospitalier que je vais m'intéresser dans cet article. Ces derniers sont, de mon point de vue, des dispositifs buissonniers de participation pour deux raisons principales.

D'abord, même s'ils sont institutionnalisés puisqu'ils doivent faire l'objet d'une autorisation par les pouvoirs publics et qu'ils sont financés par ces derniers, ces dispositifs ne

\footnotetext{
${ }^{5}$ Il s'agit de séances thérapeutiques conçues sous forme de dialogue et de travail sur soi destinées à des patients souffrant de maladies chroniques, dans le cas présent d'obésité et de diabète.
} 
correspondent pas aux dispositifs labélisés de la démocratie participative ${ }^{6}$. Il ne s'agit pas de participer à un forum citoyen, à un conseil de quartier ou à une assemblée citoyenne autour d'un projet urbain. Il s'agit plutôt de participer à des séances individuelles ou collectives de travail sur différentes thématiques (symptômes, évolution de la maladie, traitement, etc.), dans le but d'aider l'individu à prendre des décisions concernant sa propre santé et gérer au mieux sa maladie chronique. Cela suppose donc de sortir des sentiers battus de la démocratie participative en poussant la porte des cabinets médicaux, lieux d'habitude confinés et protégés des regards indiscrets. Dans ces séances de travail qui s'étendent sur plusieurs semaines, le secret médical règne : on y parle de l'intimité de l'individu, du rapport qu'il entretient avec son propre corps, de ses habitudes de vie, des difficultés psychologiques, émotionnelles ou économiques qu'il traverse et qui peuvent expliquer son mal-être ou être à l'origine d'une dégradation de son état de santé.

Ensuite, tels qu'ils sont pensés par le docteur Millot et son équipe, ces dispositifs d'éducation thérapeutique se veulent transgressifs à l'égard d'autres formes plus «traditionnelles » d'éducation thérapeutique du patient que j'ai pu observer dans le cadre de mon travail de thèse (Fauquette, 2016) et qui se traduisent, très souvent, par le maintien voire le renforcement d'un certain paternalisme médical (Elger, 2010). L'éducation thérapeutique mise en place dans le service du docteur Millot vise en effet à effacer toute forme d'imposition d'un biopouvoir (Foucault, 1976 ; Fassin, Memmi, 2004) caractéristique d'un ordre médical dominant qui s'impose aux individus de manière indirecte en valorisant l'incitation à se prendre en main, au risque de la culpabilisation (Genard, Cantelli, 2008). Ce rejet du biopouvoir s'inscrit dans un courant de pensée marginal de la prise en charge thérapeutique de l'obésité qui refuse de faire du régime et de l'observance du traitement médical une priorité, au profit de l'émancipation radicale et de l'épanouissement individuel par l'autodétermination. Au modèle dominant d'éducation thérapeutique du patient ${ }^{7}$, largement véhiculé par les pouvoirs publics en particulier la Haute Autorité de Santé qui cherche à « responsabiliser» le patient et à lui inculquer une série de «bonnes pratiques » pour qu'il change certaines habitudes de son quotidien ${ }^{8}$, s'oppose donc ce modèle émancipateur qui entend transformer profondément la relation médicale et rompre avec le biopouvoir'. Modèle qui se réclame, entre autres, de la pensée très critique d'Ivan Illich (1975) sur le pouvoir médical et trouve des accointances avec des critiques néomarxistes, féministes et post-soixante-huitardes (Zola, 1972) qui voient en la médecine une instance de contrôle social et de reproduction des rapports sociaux de domination. Modèle dans lequel l'autonomie du sujet est pensée conformément au principe du self care du début des années 1970 (Schumaker, 1977), qui renvoie à une revendication politique et sociale d'émancipation individuelle et citoyenne à l'égard des normes médicales dominantes, mais qui a été progressivement dévoyé pour être remplacé par un self management (Lorig et al., 1985 ; Lorig et al., 1999 ; Lorig et al., 2001) qui cantonne l'autonomie individuelle au respect des préceptes médicaux (Saillant, Gagnon, 1996; Klein, 2014). Modèle qui tend, dans

\footnotetext{
${ }^{6}$ D'ailleurs, l'émergence de l'ETP est bien antérieure aux succès de l'impératif délibératif ces trente dernières années : les premières expériences d'ETP apparaissent dans les années 1920 aux États-Unis, lorsqu'un jeune garçon, Léonard Thomson, fut sauvé grâce à une injection d'insuline (Grimaldi et al., 2017).

${ }^{7}$ Qui se rapproche du modèle néolibéral d'empowerment (Bacqué, Biewener, 2013).

${ }^{8}$ Il s'agit aussi, dans ce modèle, de responsabiliser les patients dans une optique de réduction des coûts liés à la santé, comme l'avaient très bien montré, dès 1972, Miller et Goldstein (1972).

${ }^{9}$ Par biopouvoir, j'entends ici, dans un sens général, «l'immixtion des pouvoirs publics ou médicaux dans la relation privée de l'individu à son destin physique », via «des technologies gouvernementales », que ce soit sous la forme d'un moralisme paternaliste ou, à l'instar des politiques de prévention aujourd'hui, dans des formes davantage incitatives (il s'agit moins d'imposer que de susciter) que l'on peut qualifier «d'incitation à la subjectivation » (Fassin, Memmi, 2004).
} 
l'absolu ${ }^{10}$, à se rapprocher du concept d'éducation à la santé et à s'opposer à celui d'éducation pour la santé, même si les limites entre les deux ne sont pas toujours très claires et qu'il existe des différences terminologiques selon les pays (Van Ballekom, 2008). Comme le soulignent en substance Geneviève Buissonnet-Verger (1997) et d'autres (French, 1990), à la différence de l'éducation pour la santé qui regroupe des pratiques hétérogènes allant de l'usage de méthodes persuasives à l'usage de méthodes motivationnelles ${ }^{11}$ visant toutes à faire adopter à l'individu un certain nombre de comportements jugés favorables pour sa santé (Green, Simons-Morton, 1984), et donc à lui faire suivre une direction particulière, l'éducation à la santé est « un processus visant l'auto-prise en charge par les individus de leur propre santé ».

Suivant ces repères, l'émancipation de l'individu telle que prônée par le docteur Millot peut donc être définie à travers deux éléments. D'une part et à nouveau, l'idée d'en finir avec la perspective selon laquelle l'institution médicale doit éduquer l'individu à certaines pratiques médicalement et socialement légitimes. En ce sens, l'émancipation consiste donc à aider l'individu à prendre conscience de la norme médicale, voire morale, qui pèse sur lui et dicte consciemment ou inconsciemment sa conduite alimentaire, et à respecter ce qu'il souhaite réellement pour le devenir de son corps et de sa santé. D'autre part, l'émancipation consiste aussi à aider l'individu à prendre conscience des autres déterminismes économiques et sociaux qui déterminent ses actions, en s'efforçant de ne pas porter de jugements moraux sur celles-ci. Toutefois, et c'est là une précaution du docteur Millot que l'on retrouve dans la pensée de Rancière au sujet de l'institution scolaire et d'autres institutions sociales, il ne s'agit pas pour autant de penser que l'institution médicale peut revendiquer une prétention à libérer les individus, autrement dit que le médecin, à l'instar des instituteurs, des artistes ou de tout autre éducateur, aurait naturellement à jouer une fonction émancipatrice. C'est ce que soutient Rancière en substance : toute démarche émancipatrice doit être pensée en fonction de cet écart par rapport à la prétention à émanciper les individus. Tout au plus, comme nous l'avons déjà souligné, le médecin ignorant, à l'instar du maître ignorant, est là pour accompagner l'émancipation des individus, mais n'est pas celui qui la permet ou la guide. Le pouvoir émancipateur ne se transmet pas mais se pratique entre égaux. C'est là une pensée qui est proche de la démarche de conscientisation de Paulo Freire (1974) pour qui « personne ne libère autrui, personne ne se libère seul, les hommes se libèrent ensemble ». C'est en effet le but de l'éducation conscientisante et dialogique qu'il prône et qui se construit à travers l'horizontalité des rapports sociaux et la communication.

Or, cette volonté d'accompagner l'individu dans son émancipation et la posture du médecin ignorant à laquelle je la rattache ici m'amènent à m'interroger sur l'une des critiques souvent adressées au cadre pédagogique de Rancière, à savoir sa difficulté d'application et son caractère trop idéaliste. À vrai dire, comme nous le verrons dans le déroulement de mon analyse, le dispositif du médecin ignorant ne s'adresse pas à tous les patients et rompt en ce sens avec l'égalitarisme ranciérien. De même, il ne parvient pas à rompre complètement avec les formes de domination médecin/patient. Dès lors, il m'apparaît heuristique de questionner le statut paradoxal et les ambiguités de ce dispositif qui se veut émancipateur : quelles sont les conditions de l'effacement relatif du pouvoir médical et ses limites ? Pourquoi ce dispositif n'émancipe-t-il que les émancipés ? Jusqu'à quel point la pratique médicale est-elle compatible avec cette posture du médecin ignorant ? Comment les patients la reçoivent-ils ? Outre ces questionnements qui me servent de fil directeur, je souhaite également interroger la

\footnotetext{
${ }^{10}$ L'éducation thérapeutique est souvent distinguée des autres types d'éducation en santé, comme le soulignent Geneviève Buissonnet-Verger (1997) et d'autres (Tessier, 2012).

${ }^{11}$ C'est notamment Jacques Bury (1988) qui distingue plusieurs approches utilisant différentes méthodes en éducation pour la santé, des plus descendantes et directes (méthodes persuasives) aux méthodes plus motivationnelles.
} 
genèse de ce dispositif buissonnier en partant de l'hypothèse que celle-ci est potentiellement traversée de tensions qui permettent aussi de comprendre les limites des prétentions émancipatrices du dispositif : comment un dispositif participatif se référant à ce point à un modèle à contre-courant du biopouvoir a-t-il pu émerger dans un espace aussi normé que celui de l'hôpital, et comment se matérialise-t-il en pratique ? Comment, et surtout sous couvert de quel(s) compromis, cet espace alternatif a-t-il pu pousser en secret et en marge de l'institution hospitalière?

Dans une première partie, j'étudie la genèse de cette entreprise participative en lien avec la socialisation du docteur Millot et identifie quelques éléments qui la rendent possible, ainsi que certaines de ses limites. Dans une seconde partie, j'aborde les lignes de fuite et les paradoxes de cette pratique alternative, ainsi que les réceptions différenciées qu'en ont les patients.

\section{Encadré 2. Le terrain d'enquête et les méthodes utilisées}

Dans le cadre de ma thèse en science politique dédiée à l'étude de la démocratie sanitaire, je me suis rapidement rendu compte que les agents de mes différents terrains utilisaient souvent le terme de participation des publics pour parler d'autre chose que de démocratie participative. Il s'agissait non pas de la participation de l'individu à des dispositifs labélisés de démocratie participative, mais de la participation de l'individu à sa propre santé, ou encore de la participation du patient à la consultation médicale. C'est en décidant de prendre au sérieux ces conceptions émiques de la participation que je me suis alors intéressé à certains dispositifs auxquels ces conceptions pouvaient renvoyer. Or, parmi ces dispositifs, il y avait, entre autres, ces dispositifs d'éducation thérapeutique du patient qui visent à rendre le malade «acteur de sa santé ». C'est donc avec les yeux d'un jeune chercheur habitué aux travaux sur la démocratie participative que je me suis intéressé à l'ETP durant mon travail doctoral, ou aux éducations en santé de manière plus générale, en cherchant à faire dialoguer les travaux de sociologie de la santé qui lui sont consacrés (Flora, 2013 ; Tourette-Turgis, 2015), avec les travaux sur la participation, en particulier les travaux s'inscrivant dans les théories du contrôle participatif (Gaxie, 1996 ; Jouve, 2005) et plus largement les travaux s'inscrivant dans le champ de la sociologie critique de la participation.

J'ai rencontré le docteur Millot en juin 2014 au cours d'un séminaire sur l'ETP. À la suite de nos premiers échanges, il m'a autorisé à observer l'ensemble des séances d'ETP de son service. J'ai ainsi pu suivre, courant 2015, les six séances de son dispositif via des observations participantes non enregistrées. Chaque séance durait environ une heure trente et portait sur différentes thématiques comme les représentations sociales de l'alimentation et du corps, la dégustation, l'expérience de la faim. S'ajoute à ces observations participantes la tenue de plusieurs entretiens semi-directifs : quatre entretiens avec le docteur Millot, trois entretiens avec deux de ses collègues, une médecin nutritionniste et un diététicien, et quatre entretiens avec des participants aux différentes séances ${ }^{12}$. En ce qui concerne mes entretiens avec les professionnels de santé, mes questionnements ont porté sur les raisons qui les ont amenés à vouloir exercer leur profession et la manière dont ils considèrent leur rappors au patient. Une partie de ces entretiens a porté sur les rapports qu'ils entretiennent avec le reste du corps médical. En ce qui concerne les patients, je les ai interrogés sur leur parcours de santé, les ressorts de leur participation et ce qu'ils ont retiré de cette expérience. En plus de ces entretiens formels avec l'équipe médicale ou avec certains patients, $\mathrm{j}$ 'ai multiplié les temps de discussion informelle, notamment lorsque nous rentrions à nos domiciles respectifs à la fin de chaque séance.

Il est à souligner que le dispositif d'ETP du docteur Millot n'est pas le seul dispositif que j'ai étudié dans ma thèse. J'ai en effet observé deux autres dispositifs d'ETP, l'un en milieu hospitalier et l'autre en milieu libéral. Or, si j'ai choisi dans le présent article de focaliser essentiellement mon attention

\footnotetext{
${ }^{12}$ J'ai aussi interrogé 7 autres patientes qui participent à un dispositif de thérapie comportementale sur les troubles des conduites alimentaires.
} 
sur le dispositif du docteur Millot, c'est qu'il m'est apparu original par rapport aux deux autres dispositifs, tant du point de vue de son cadre de pensée qui pose clairement la question de l'émancipation de l'individu, que du point de vue des interactions sociales qui se nouent durant chacune de ses séances. Mais ce dispositif m'est également apparu original du fait des particularités de la question du surpoids qu'il aborde et qui influe à la fois sur le mode de recrutement des participants et sur les interactions sociales entre professionnels de santé et patients. En effet, contrairement à des maladies chroniques clairement diagnostiquées par le corps médical ${ }^{13}$ comme devant faire l'objet d'un suivi médical plus ou moins urgent, intensif et régulier, le surpoids relève davantage d'une prise de conscience par l'individu lui-même de ce qu'il estime être son surpoids. De fait, une bonne moitié des patients du docteur Millot interrogés révèlent être venus d'eux-mêmes au Cétrabète, ou sur les conseils d'un proche, parce qu'ils se disent «mal dans leur peau» et/ou soucieux de leur apparence physique. Il s'agit donc d'un public qui est déjà potentiellement conscientisé et/ou en voie d'émancipation.

\section{Une volonté de rompre avec l'asymétrie de la relation médicale}

La mise en place de cette ETP que j' ai qualifiée de buissonnière, c'est d'abord la volonté d'un chef d'équipe qui a fait des émules et recruté un personnel en fonction de sa disponibilité à entendre son discours émancipateur et égalitariste ${ }^{14}$. Il nous apparaît donc important d'apporter quelques éléments sur la socialisation de cet individu.

\section{Le médecin ignorant}

Loin des «bulldozers de certitudes », comme il aime à qualifier certains médecins qui imposent à leur patient des vérités médicales de manière implacable, cet admirateur de la pensée d'Edgard Morin, d'Illich ou encore de Foucault, assume très ouvertement un positionnement à l'extrême gauche de l'échiquier politique. Proche à la fois de la pensée libertaire et du mouvement des Insoumis, il s'inscrit dans l'héritage des mouvements alternatifs de médecins qui émergent au cours des années 1970 (Hassenteufel, 1997). Fils d'enseignants communistes syndicalisés à la CGT, il bénéficie déjà d'un capital culturel et politique important lorsqu'il commence ses études de médecine. Mais il est au départ ce qu'il appelle un étudiant ordinaire qui choisit ses options un peu "par hasard» et n'a pas nécessairement de positionnement clair sur la médecine et la politique, surtout qu'il ne se reconnaît pas dans le conservatisme de la critique sociale du capitalisme (Boltanski, Chiapello, 1999) dans lequel s'inscrit son père. C'est progressivement qu'il adopte un positionnement critique à l'égard du pouvoir médical et peaufine son positionnement professionnel et politique, sous l'influence de trois individus.

Il y a d'abord son oncle, un ancien médecin devenu grand ponte de la sécurité sociale, qui fait pour lui figure de «modèle » pour sa vision systémique des questions de santé, et qui, le premier, lui a fait entendre un discours très critique sur le pouvoir médical et la médecine libérale.

Il y a ensuite une ex-petite amie, une bibliothécaire de vingt ans son aînée qui, selon lui, lui a transmis un esprit de bienveillance à l'égard d'autrui : "C'était quelqu'un qui m'acceptait entièrement tel que j'étais ». Esprit que l'on retrouve dans sa pratique médicale quotidienne qu'il caractérise, en référence à Carl Rogers (1952), de «médecine centrée sur la personne », à savoir une médecine qui suppose une écoute attentive et empathique du patient et un non- 
jugement que Rogers qualifie de « regard positif inconditionnel». Posture de non-jugement que le docteur Millot s'efforce continuellement de maintenir et qui s'inscrit en porte-à-faux avec ce qu'il qualifie lui-même de "glissement anthropologique », à savoir une « obligation de s'occuper de soi, de se prendre en main, de se responsabiliser». Posture difficile à tenir dans la mesure où la tentation de juger est grande, notamment lorsqu'il fait face à des patients dont il ne comprend pas le comportement. Seule la posture de non-jugement du professionnel de santé, couplée avec un profond respect du principe d'égalité avec le patient permet, selon lui, l'émancipation de l'individu: "Cette posture du professionnel rend possible le changement, le choix ». C'est d'ailleurs ce que souligne Rogers pour lequel «le thérapeute éprouve une attitude positive, non jugeante et d'acceptation face à tout ce que le client est en ce moment (peu importe ce qu'il est à ce moment-là), il est vraisemblable qu'un mouvement ou changement thérapeutique se produira ».

Il y a enfin un membre de sa famille que le docteur Millot appelle le «fou libre » et qu'il admire pour son esprit et son mode de vie libertaires : "C'est quelqu'un qui se défait des codes sociaux, qui fait ce qui lui plaît et c'est fascinant, il n'a pas de pesanteur sociale sur lui ». Là aussi, le docteur Millot y trouve l'une des sources d'inspiration de sa pratique médicale libertaire. Pratique qui, selon lui, va bien plus loin que le précepte «mangez à votre faim et à votre satiété » porté par certains groupes de réflexion, à l'instar du GROS (Groupe de réflexion sur l'obésité et le surpoids), des fat studies ou du mouvement Health at Every $\mathrm{Size}^{15}$. Certes, ces mouvements remettent en cause l'impératif moral de perte de poids porté par une grande partie du corps médical à l'endroit des individus en surpoids, et visent à redonner à ces derniers le droit d'être ce qu'ils sont, à savoir des individus dignes. Ceci étant, selon le docteur Millot, ces mouvements cultivent en même temps une certaine ambiguïté : l'idée de perte de poids demeure un objectif implicite ou inconscient, et la bienveillance ainsi que la lutte contre la stigmatisation un moyen pour y parvenir. Or, selon lui, la perte de poids n'est pas un objectif, encore moins une direction à suivre. Les seuls objectifs sont ceux que se fixe l'individu.

Mais ce qui le différencie, peut-être plus encore, de ces courants de pensée s'inscrivant déjà à la marge de la normativité médicale, c'est surtout son attachement au principe d'égalité entendu dans un sens ranciérien :

« Pour moi, l'égalité est un point de départ et non un point d'arrivée. C'est mon postulat visà-vis du patient, une sorte d'égalité ontologique qui suppose une très grande exigence, un peu comme la liberté chez Sartre. »

Cette exigence d'égalité détermine sa pratique médicale qu'il qualifie de restructuration cognitive et qui s'objective par une série de questions ouvertes favorisant l'attitude de nonjugement du thérapeute, ou ce que Rogers qualifie de regard positif inconditionnel (1980) :

«Ce n'est rien d'autre que de la restructuration cognitive: "Vous dites cela, vous pensez cela, mais qu'est-ce qui fait que vous vous dites cela, qu'est-ce qui fait que vous pensez

\footnotetext{
${ }^{15}$ Il s'agit de courants interdisciplinaires et militants, apparus aux États-Unis et peu connus en France, qui dénoncent les discriminations et risques de culpabilisation des personnes vivant en surpoids.
} 
cela ?" [...]. Ce n'est pas de dire aux gens "C'est de votre faute, prenez-vous en main, devenez acteurs !" [...] Comme s'ils avaient à devenir acteurs. » ${ }^{16}$

Il convient de souligner que cette exigence d'égalité fortement théorisée dans le discours du docteur Millot n'est pas partagée par l'une de ses collègues nutritionnistes beaucoup moins politisée que lui. Bien qu'elle affirme pratiquer elle-même la démarche de restructuration cognitive dans ses consultations médicales, elle ne semble pas conquise par la posture du médecin ignorant, étant attachée à son savoir biomédical.

\title{
Amoindrir la distance sociale et cultiver le «lâcher-prise »
}

Toujours est-il que cette exigence d'égalité n'est pas qu'un argument rhétorique formulé par un docteur en marge du corps médical, mais s'observe très concrètement dans les pratiques d'ETP de son équipe médicale qui s'efforce, autant que faire se peut, d'amoindrir la distance sociale avec les patients en cultivant le lâcher-prise. Cet effort se matérialise tout d'abord dans une mise en espace particulière qui tend à démédicaliser les rapports sociaux. Le paragraphe ci-après, tiré d'une note de ma première observation participante, en témoigne :

\begin{abstract}
« Nous sommes dans un service hospitalier situé dans une vieille bâtisse à l'extérieur de l'hôpital. Les lieux sont imposants et respirent l'histoire : le couloir est recouvert d'un vieux parquet craquant qui donne sur un large escalier en pierre, dont les murs sont ornés de tableaux gigantesques qui se confondent avec les moulures du plafond. Seuls quelques chaises métalliques et des magazines aux pages partiellement déchirées nous rappellent que nous sommes dans un couloir de salle d'attente. À part ces éléments, rien ne laisse présager que nous nous situons dans un lieu médical, et encore moins dans un service hospitalier, si ce n'est que certains professionnels de santé traversent parfois le couloir vêtus d'une blouse blanche. On se croirait plutôt au siège social d'un parti politique ou d'une "grande" association. L'ETP est délivrée dans une salle à l'aménagement non moins surprenant pour une séance dite éducative : pas de tables, si ce n'est en fond de scène et posées les unes sur les autres pour les utiliser "au cas où", mais des chaises positionnées en rond, comme dans des groupes de parole. En ce sens, les regards des participants ne sont pas projetés dans une direction univoque, celle qui place habituellement le professionnel de santé au centre des attentions, mais dirigés à parts égales sur chacun des participants. Le professionnel de santé est d'ailleurs assis, comme les autres participants, sur une chaise, et j'ai au départ quelque difficulté à le distinguer. De très grandes baies vitrées offrent une vue imprenable sur un jardin aux arbres grandioses et aux allées fleuries. Tout est fait pour cultiver la détente et le "lâcher-prise", échanger à tête reposée dans une atmosphère calme et hors du temps. La majestuosité des lieux donne à chacun une hauteur de vue [...]. Comme l'affirme une patiente quelques minutes après notre arrivée: "Quand on entre au Cétrabète, on n'a pas l'impression que l'on va nous juger. On est bien". »
\end{abstract}

Contrairement aux autres dispositifs d'ETP que j'ai pu observer, je n'ai pas l'impression d'entrer dans une salle de cours pour y suivre un enseignement magistral, mais d'entrer dans un lieu où la distance sociale entre le professionnel de santé et les patients se trouve réduite, sans pour autant que le pouvoir médical disparaisse. Deux éléments semblent être ici assez significatifs. Il y a d'abord la démédicalisation du lieu qui tend à effacer les signes 
ostentatoires du pouvoir médical. Il y a ensuite la position du professionnel de santé qui, assis dans le cercle au même titre que les autres participants, n'est pas dans la position explicative des «monopoleurs d'intelligence assis sur le trône explicateur» dont parle Rancière lorsqu'il fait référence aux maîtres d'école. Certes, au cours des séances suivantes, il lui arrive parfois de se lever pour prendre des notes sur un paperboard ou pour préciser sa pensée. Mais je constate au cours des six séances que ces moments sont assez rares et que le professionnel de santé s'efforce de se placer à la même hauteur que ses patients : «Je ne souhaite pas donner l'impression de faire un cours magistral, ce qui suppose de me mettre dans la même position qu'eux autant que possible. » Il s'agit donc bien d'un effort pour horizontaliser les rapports sociaux sachant que, comme le souligne Foucault (1994), l'éradication des relations de pouvoir est une tâche irréalisable.

Les échanges eux-mêmes témoignent de cette volonté d'amoindrir la distance sociale entre le professionnel de santé et les patients. L'encadré ci-après, qui est la suite de la note précédente, en témoigne.

\section{Encadré 3. Des échanges équilibrés}

Le professionnel de santé, Monsieur Piette, est un jeune diététicien qui, grâce à son humour et ses tenues vestimentaires décontractées (il met souvent un jean et des baskets de ville), installe un climat de confiance et de détente apprécié de la plupart des participants : beaucoup le disent à chaque début de séance, et la plupart reviennent emplis de bonne humeur et, comme le dit Sophie, professeur d'espagnol, «d'une envie de partager ». Le message qu'il répète ici et là à ses patients depuis le début de l'ETP nous apparaît désormais de plus en plus clair: "Se faire confiance ». Un tel message présente l'avantage de «briser » certains sujets tabous, comme le rapport des personnes à leur corps ou encore les discriminations dont elles ont été victimes en tant que personnes en surpoids.

Le diététicien.— « Alors, comment avez-vous trouvé la dernière séance ?

Un patient.- On est sortis apaisés, on parlait sans pression, sans complexe. On se sent beaucoup moins seuls.

Le diététicien.- Vous n'avez pas eu de difficultés particulières?

Le patient._- Oui, ça n'était pas compliqué à comprendre [...]. Après... (il réfléchit longuement avant de poursuivre son propos, donnant l'impression de peser l'ensemble de ses mots tant son idée semble complexe), après ça n'est pas aussi... Enfin, je veux dire que, présenté comme cela, comme vous l'avez fait, on a l'impression que c'est au bout d'un long moment, d'un long processus que la frustration et la culpabilité émergent [...]. La culpabilité est un sentiment qui arrive à la fin, et résulte d'une pression sociale extérieure, au travail ou ailleurs. On croit devoir assumer une responsabilité, d'une chose qui ne relève finalement pas de nous, et c'est très destructeur.

Le diététicien.- Oui, on va d'ailleurs être amenés à réfléchir ensemble à la temporalité entre les différentes phases que sont le régime, la frustration, le craquage et la culpabilité. »

Une patiente lui coupe la parole, inspirée par une idée soudaine et fulgurante : «Moi, j'ai banni tous les interdits cette semaine, comme vous nous l'aviez conseillé. Et ça m'a redonné le moral.

Le diététicien.- Du coup, ça vous crée une forme d'euphorie c'est ça ? 
La patiente.- Je ne sais pas si c'est cela, ou si ça n'est pas plutôt, finalement, une forme de détente. On lâche prise avec la pression sociale, on se fait confiance en ne s'interdisant pas de se faire plaisir et finalement on se contrôle mieux, on se connaît davantage et on se remet à sourire. »

Une autre patiente intervient : "Oui, c'est vrai. On se pose moins de questions, mais en même temps, on a d'autres questions qui nous viennent en tête, comme celle de se dire : "C'est bien, j'ai mangé, je me suis fait plaisir, mais... est-ce que je ne vais pas prendre du poids ?"

Le diététicien.- Oui, je comprends. Et vous vous dites: "Le diabétologue m'a dit que je pouvais tout manger", mais "Qu'est-ce que ça va donner?" Je vous le dis de suite : je ne détiens pas la vérité, on n'en sait rien, et on verra bien ce qui se passe !»

Quelques minutes plus tard, une patiente s'exprime de sa propre initiative sur son addiction au chocolat : "Pour moi, le chocolat, ça sert à me remplir quand ça ne va pas, dans ces moments-là, je ne gère plus rien. » Ce à quoi le professionnel répond : « On a le droit de ne pas gérer, on a le droit de lâcher prise [...]. Je ne sais pas ce qui est bon ou pas, je ne place quasiment rien dans les interdits [...]. "C'est à vous de décider". » L'objectif principal de l'ETP n'est donc pas la perte de poids. Il s'agit pour les participants de se réapproprier leur alimentation, sans limitation abusive mais avec un droit manifeste et revendiqué au lâcher-prise.

Deux éléments principaux attestent de cet effort pour équilibrer les échanges sociaux. Il y a, à nouveau, la posture d'humilité que tient le professionnel de santé qui, à travers ses propos, refuse ouvertement de jouer le rôle du savant ou du prêcheur moral et rappelle à ses patients leur pouvoir de décision. Loin de donner l'impression de détenir «la » vérité, ou plutôt une vérité médicale érigée en croyance ${ }^{17}$ qui n'aurait plus à se démontrer, il semble chercher les réponses à ses propres doutes dans le discours des patients, les interrogeant sur leurs ressentis, se mettant lui-même dans le rôle de celui qui doit apprendre pour aider s'il le peut, qui accompagne la réflexion mais ne juge pas, qui n'a pas le pouvoir ni la volonté de changer les autres, qui ne joue pas de son influence ou du pouvoir que lui confère son statut. Il rompt avec le postulat explicatif, en avouant lui-même qu'il ne sait pas, et préfère celui de l'accompagnement de la réflexion individuelle. Il est celui qui insuffle un esprit de liberté dont les participants n'hésitent pas à se saisir.

C'est justement là le deuxième élément que la note met en évidence : dans mes observations, les patients semblent se conformer parfaitement à cette situation particulière dans laquelle le pouvoir médical cherche à s'effacer. Ces derniers parlent longuement, parfois davantage que le professionnel de santé, n'hésitant pas à lui couper la parole quand une idée fulgurante leur traverse l'esprit, à l'instar de la patiente évoquant son rapport aux interdits. En somme, ce sont les patients qui donnent le tempo de la discussion. Ces patients qui, par ailleurs, ne rechignent pas à donner leur point de vue sans que le professionnel le leur ait demandé, ou à apporter des précisions aux propos de ce dernier. Certains vont même jusqu'à exprimer leur désaccord et font preuve d'un esprit critique qui ne se tarira pas au cours des différentes séances. C'est même le contraire qui se produit: par la répétition des rencontres, chaque participant parle de plus en plus facilement des différentes pressions sociales (y compris celles

\footnotetext{
${ }^{17}$ Monsieur Piette prend plaisir à jouer ce rôle du professionnel de santé ignorant et à multiplier ce qu'il estime être des dissonances cognitives : «Ce qui m'éclate, c'est de jouer l'ignare [...]. De dire aux patients : "Je ne sais pas, dites-moi ce que vous en pensez". J'adore créer de la dissonance ».
} 
de certains professionnels de santé «culpabilisateurs ») dont il a été victime et des moyens qu'il a trouvés ou inventés pour y résister.

\section{Une dynamique émancipatrice?}

$\mathrm{Si}$, comme on le verra dans la suite de notre analyse, ce dispositif rate sa cible et n'émancipe qu'un public averti, il n'en reste pas moins qu'on peut observer une vraie dynamique émancipatrice pour trois raisons principales.

D'abord, c'est à l'individu qu'il incombe de fixer ses propres règles ${ }^{18}$, de se réapproprier son corps, de mieux se connaître, sans qu'un objectif extérieur, qui ne serait éventuellement pas le sien, vienne troubler ce processus. Mais ce contrôle n'est pas infaillible : il ne s'agit pas de faire des individus des sortes d'automates qui contrôleraient entièrement leurs besoins et leurs désirs. Il y a au contraire, comme nous l'avons souligné précédemment, une revendication manifeste à l'imperfection et au droit de «lâcher prise ». Il s'agit surtout de sortir de la culpabilisation et de gagner de la confiance en soi. Bref, de gagner en liberté de gestion de son corps.

Ensuite, les échanges permettent des montées en généralité opérées par les participants pour décrire leur situation individuelle. On évoque à tour de rôle les pressions sociales exercées sur les individus en surpoids, les dérives de la société de consommation qui sollicitent les individus à consommer plus tout en les faisant culpabiliser, la stigmatisation et les discriminations dont chacun se dit victime. Les anecdotes utilisées par chacun servent à éclairer un ensemble de processus sociaux et de variables qui pèsent sur les individus en surpoids. En parlant de sa situation individuelle, l'individu parle aussi pour les autres et au nom des autres par ses montées en généralité. Il s'agit ici d'une forme de politisation ${ }^{19}$ des questions de santé qui, tout en évitant la question du politique stricto sensu, tend à conférer aux individus un esprit critique sur le fonctionnement de la société et du système de santé.

Enfin, la dynamique émancipatrice s'observe aussi dans la prise de recul de l'individu avec ses propres certitudes à l'égard de la nourriture. Par exemple, il n'y a pas d'un côté de « bons » aliments et de l'autre de «mauvais » aliments, comme le démontre notre observation participante ci-après tirée d'une séance dédiée à la dégustation :

« Nous assistons à une séance de dégustation où chacun découvre ou plutôt redécouvre les aliments en décrivant longuement ce qu'il/elle ressent. Nous devons fermer les yeux et sentir, toucher, puis goûter différents types de chocolats et décrire ce que nous ressentons. Les surprises sont de taille: certains qui prétendaient préférer le chocolat blanc découvrent des chocolats noirs bien meilleurs, d'autres qui prétendaient que le chocolat noir est moins gras que le chocolat blanc aboutissent à la conclusion inverse, etc. Bref, notre esprit se brouille et nous ne sommes plus sûrs de rien, si ce n'est que les interdits sont souvent nuisibles et que l'on se fait souvent de fausses idées sur les aliments. »

\footnotetext{
${ }^{18}$ Non pas celles qui fixent le cadre de la réunion mais celles qui concernent sa propre santé.

${ }^{19}$ J'identifie ici la montée en généralité des propos de l'individu comme élément de politisation de son discours (Hamidi, 2006). Cette politisation tend à réduire la frontière entre les conceptions individualisées et collectives de la participation puisque c'est de l'expérience individuelle et de son expression que s'opère la montée en généralité.
} 
L'observateur que je suis se trouve donc lui-même pris au jeu de cette expérience surprenante ${ }^{20}$ qui déconstruit totalement nos a priori et nous permet d'apprécier davantage le goût des aliments. Cette dynamique émancipatrice se repère tout particulièrement lors de la dernière séance d'ETP, lorsque les participants sont invités à remplir un tableau dans lequel ils formalisent leur projet individuel, les progrès ressentis ou les difficultés éprouvées au cours des séances, et leur situation actuelle. Le registre de la prise de conscience, de la prise de recul et du changement personnel sont alors très utilisés, comme en témoigne ci-après la reproduction fidèle de trois cas répertoriés dans ce tableau. 
Tableau 1. Les effets de l'ETP sur les individus

\begin{tabular}{|c|l|l|l|}
\hline Prénom & \multicolumn{1}{|c|}{ Progrès } & \multicolumn{1}{|c|}{ Difficultés } & \multicolumn{1}{c|}{ Et maintenant ? } \\
\hline Vanessa & $\begin{array}{l}\text { Moins de culpabilité. } \\
\text { Prise de recul sur cette } \\
\text { culpabilité destructrice. } \\
\text { Mange quand elle a faim. }\end{array}$ & $\begin{array}{l}\text { Lutter contre l'ennui n'est } \\
\text { pas simple. }\end{array}$ & $\begin{array}{l}\text { Besoin puive } \\
\text { psychologiquement. Une prise } \\
\text { de recul lui en a fait prendre } \\
\text { conscience. }\end{array}$ \\
\hline Fatima & $\begin{array}{l}\text { Prise de conscience de la } \\
\text { nécessité d'arrêter de se } \\
\text { faire du mal et de la } \\
\text { nécessité d'être davantage } \\
\text { à l'écoute de son corps. }\end{array}$ & $\begin{array}{l}\text { Projet de devenir animatrice } \\
\text { sportive, de prendre soin de soi } \\
\text { et des autres. }\end{array}$ \\
\hline Sylvie & $\begin{array}{l}\text { S'est arrêtée de } \\
\text { s'interdire. } \\
\text { Changement d'objectif } \\
\text { personnel désormais, se } \\
\text { donner des objectifs } \\
\text { réalisables. }\end{array}$ & $\begin{array}{l}\text { Encore des difficultés à } \\
\text { gérer son stress et ses } \\
\text { Prise de conscience de } \\
\text { l'utilité de s'écouter } \\
\text { davantage }\end{array}$ & émotions. \\
\hline
\end{tabular}

Ce tableau mérite toutefois d'être nuancé dans la mesure où il est produit à la fin d'une séance d'ETP, ce qui favorise un certain entre-soi et un phénomène de mimétisme, les participants reprenant parfois à leur compte les propos de leurs voisins. Par ailleurs, le fait que le dispositif participatif est un dispositif de soin qui porte sur une maladie chronique limite son potentiel émancipateur. En effet, dans le cas des maladies chroniques, l'autonomie du patient est une forme de dépendance maîtrisée: il s'agit pour le malade d'être accompagné par les professionnels de santé pour pouvoir vivre quotidiennement avec sa maladie (Ricœur, 1990, 2004), son expérience sensible. De sorte que l'autonomie des malades n'est rendue possible que par leur dépendance aux professionnels de santé. Être autonome, nous dit Céline Lefève (2015) «ne signifie pas être indépendant car seul, mais conscient de sa dépendance et bien accompagné ».

Chez certains participants de cet ETP, cette dépendance semble être paradoxalement une dépendance au discours déculpabilisant et à la posture du médecin ignorant. Compte tenu de la dimension anxiogène des questions de santé, le discours déculpabilisant des professionnels de santé peut en effet créer une dépendance au discours d'émancipation, comme un besoin d'être rassuré d'autant plus fort qu'il touche au corps de l'individu, à son intimité. Il constitue en ce sens un facteur de participation potentiel. C'est le cas de Mariana qui, à la fin d'une séance, nous dit en substance : 
«Je ne pourrais plus aller voir d'autres professionnels de santé, ici on est écoutés, respectés et donc rassurés. Ils ne nous culpabilisent pas, ça fait un bien fou [...]. Ils ne nous regardent pas en nous disant ce qu'il faut faire d'un air sévère [...]. C'est difficile à vivre le regard des autres sur notre corps et là, bah, c'est un vrai échange, ils ne sont pas dans l'état d'esprit "c'est moi qui sais, toi tu te tais et tu fais ce que je te dis de faire, arrête de te goinfrer" [...]. J'ai besoin d'eux. Je n'ai jamais vu ça ailleurs, c'est très particulier. »

\section{Des conditions favorables}

La dernière phrase de Mariana nous amène à nous questionner à présent sur la genèse de ce dispositif «très particulier ». Comment un dispositif émancipateur refusant à ce point la doxa responsabilisatrice et les atours du pouvoir médical a-t-il pu voir le jour au sein d'un hôpital ? Grâce à plusieurs conditions particulièrement favorables.

La première de ces conditions, c'est d'abord et à nouveau le «type » de maladie ${ }^{21}$ que traite cette ETP. On le sait, quand il est atteint d'une maladie chronique, c'est-à-dire d'une maladie qui dure dans le temps et que l'on ne sait «ni prévenir, ni guérir » (Grimaldi et al., 2017), le malade doit presque «toujours participer activement à son traitement », " acquérir un savoir et même, dans certains cas, la maîtrise de techniques complexes qu'il ne doit pas craindre d'appliquer à son propre corps » (Adam, Herzlich, 2012). Le traitement d'une maladie chronique nécessite donc de la part du corps médical de porter une attention toute particulière à l'environnement social de la personne, à son mode de vie et aux dimensions socioéconomiques qui structurent son existence (Canguilhem, 1994). Ce qui tend à faire évoluer la relation médicale dans un sens plus collaboratif et moins prescriptif. Or, l'obésité est certainement l'exemple même de la maladie chronique poussée à son paroxysme : qualifiée parfois de maladie urbaine (Larchet, 2015) ou de maladie du pauvre, son traitement médical est souvent incertain, en témoigne la très grande variété des régimes alimentaires dont les limites sont souvent pointées du doigt (Fischler, 1990 ; Régnier, 2010). L'incertitude et l'ignorance médicale dominent d'autant plus lorsque l'obésité de l'individu est causée par des addictions alimentaires. Comme l'affirment Clarisse Gervais, Mario Sanchez et William Lowenstein (in Bergeron, Castel, 2010) à ce sujet :

«Pourquoi sont-ils [les patients] donc si réticents à éviter les aliments gras, salés, et à manger cinq fruits et légumes ? C'est parce que nous ne parvenons pas à mieux détailler et à expliquer ce qui arrive au patient, dans notre marécage d'ignorance, que se propage l'idée que ce dont il souffre est un trouble qui dépasse sa "volonté", faute de discréditer notre savoir [...] Nous ne parvenons même pas à nous dire, médecins, psychologues, scientifiques et société, que nous sommes pour ce qui est des addictions dans l'ignorance propre aux maladies qui nous échappent, totalement. »

Par ailleurs, l'obésité est au creuset du sanitaire et du social (Poulain, 2009) et n'engendre que très rarement d'urgence médicale. C'est là une différence majeure entre l'obésité et d'autres maladies chroniques, comme l'insuffisance rénale par exemple, qui est davantage marquée

\footnotetext{
${ }^{21}$ Lorsque je parle de «la maladie » au sens générique du terme, je ne parle pas d'une réalité organique, mais plutôt d'un construit social. Voir Goffman (1979), Strauss (1992), Barrett (1999), Huyard (2012).
} 
par l'incertitude quant à l'évolution de la maladie ${ }^{22}$. Cette absence d'urgence médicale est un atout majeur pour le développement des pratiques émancipatrices ${ }^{23}$ du docteur Millot, comme il le dit lui-même :

«Ici, nous n'avons jamais d'urgence médicale, donc on peut se permettre de prendre le temps de penser l'émancipation des individus avec eux, de ne pas être dans un registre paternaliste et descendant. Il ne s'agit pas de sauver un malade là, de sauver une vie [...] Je suis bien confortable avec cet objet de l'obésité qui est un magnifique objet pour dépasser le paradigme biomédical [...]. On a le temps de travailler le fond des sujets, d'aller au fond des choses avec les patients. »

La dernière phrase de cette citation est particulièrement éclairante : ces professionnels de santé ont souvent l'impression de ne plus faire de la médecine, mais de l'accompagnement au développement psychologique et social de l'individu. À vrai dire, ils ne touchent presque jamais le corps de leurs patients et n'ont pas à faire d'interventions médicales stricto sensu. Ce qui facilite le contournement du pouvoir médical puisque celui-ci est, entre autres, un pouvoir physique (au sens propre du terme) du médecin sur le corps de son patient. Pouvoir qui place automatiquement ce dernier en position de subordination. De fait, l'absence de ce pouvoir direct sur le corps du patient permet à ces professionnels de santé d'inscrire plus facilement la relation médicale sur un mode horizontal.

Mais ce sont aussi des éléments structurels liés à leurs professions qui permettent aux professionnels du Cétrabète de peaufiner leur ETP autour de la posture du médecin ignorant. Le fait d'exercer une activité salariée leur confère notamment un rapport au temps beaucoup plus souple et des marges de manœuvre plus importantes que des professionnels de santé libéraux. Là où, en milieu libéral, des programmes d'ETP sont souvent fournis clés en main aux professionnels de santé dans un objectif de "gain de temps », l'équipe du Cétrabète a pu prendre le temps de construire son propre programme, selon ses propres orientations éthiques et philosophiques. Comme le souligne le diététicien en charge de l'animation de l'ETP :

«C'est un programme construit par l'équipe, mûrement réfléchi, que l'on fait d'ailleurs toujours évoluer en fonction de nos échanges les uns avec les autres [...]. On a vraiment des marges de manœuvre que l'on n'aurait pas en tant que libéral. C'est un climat, une équipe. »

La tarification à l'activité et la recherche constante de rentabilité économique qu'elle induit au sein de l'hôpital public n'annihilent pas ces avantages du salariat. Elle permet même de financer une partie des dispositifs buissonniers du Cétrabète, pour peu que le budget du service reste à l'équilibre. C'est en effet grâce à la multiplication des activités à forte valeur

\footnotetext{
${ }^{22}$ Cette différence peut avoir un double effet : à la fois sur l'attitude des professionnels de santé, et sur le degré d'autonomie et d'empowerment des individus. D'un côté, l'insuffisance rénale renforce la dépendance de l'individu au corps médical et aux machines alors que, d'un autre côté, la gestion de l'obésité peut se faire dans un rapport plus distancié.
} 
ajoutée, à l'instar des hospitalisations ou des interventions chirurgicales, que le docteur Millot dote son ETP de moyens supplémentaires à ceux qui lui sont déjà alloués par l'État ${ }^{24}$.

Les professionnels du Cétrabète bénéficient aussi de marges de manœuvre très importantes pour penser et concevoir leur ETP, à l'abri du regard des pouvoirs publics. Marges de manœuvre que ne réduit pas l'institutionnalisation du dispositif, bien au contraire. L'institutionnalisation sert en effet la posture transgressive du médecin ignorant et objective son caractère buissonnier. Plusieurs raisons peuvent ici être avancées.

Certes, le programme d'ETP du Cétrabète doit obligatoirement, à l'instar de tous les programmes d'ETP, être soumis à la validation puis à l'évaluation des instances de l'ARS. Pour ce faire, le docteur Millot est contraint de remplir un dossier type comportant un certain nombre de données (nombre de participants et de professionnels de santé engagés, type de pathologie, thématique de chaque séance, etc.), ainsi qu'un rapport d'activité annuel. Mais ces dossiers types, tout aussi formels qu'ils puissent être, n'en demeurent pas moins très sommaires et éloignés de la réalité des pratiques. Les questions posées sont majoritairement des questions fermées pour permettre aux professionnels de santé de répondre facilement et rapidement. Il ne s'agit aucunement de décrire la philosophie du dispositif, ni les manières d'entrevoir la relation médicale ou la posture du professionnel de santé. Par ailleurs, aucune évaluation in situ ne s'opère, ni aucun audit. Autrement dit, une fois que le programme d'ETP est accepté administrativement par les pouvoirs publics, le professionnel de santé est seul face à ses patients et guide l'ensemble de la procédure comme bon lui semble ${ }^{25}$. Le politique est alors absent, invisible.

L'institutionnalisation du dispositif ne s'oppose donc pas au buissonnier, bien au contraire : c'est dans les points aveugles de l'institutionnalisation que l'on trouve le buissonnier. Comme le souligne le diététicien : «Ils [les pouvoirs publics] ne savent pas réellement ce que l'on fait, on peut faire ce que l'on veut sans qu'ils sachent, mais en même temps je dirais qu'ils s'en moquent ». L'impression demeure effectivement, au sein de l'équipe du Cétrabète, que les pouvoirs publics, ainsi que la direction hospitalière, n'accordent pas beaucoup d'importance à l'ETP dans la mesure où l'enjeu central de l'offre de soins hospitalière est devenu, depuis la réforme sur la tarification à l'activité de 2007 (Mas, Pierru, Smolski, 2011), la rentabilité économique. Comme le précise le docteur Millot :

«Les questions liées à la posture médicale ou à la relation de soin, ce n'est pas le sujet de ma direction hospitalière. Leur première priorité, et ils ne manquent pas de nous le rappeler, c'est que la marge financière de l'activité (différence recette/dépenses) progresse. La qualité des soins et la satisfaction des patients sont des moyens pour atteindre cet objectif. L'ETP n'est pour eux qu'un enjeu financier mineur, peut-être surtout un enjeu marketing. Pour ma part, j'ai eu la possibilité de renforcer mon équipe car je me suis engagé à faire plus d'hospitalisations, et aujourd'hui, on me laisse tranquille parce que je suis bénéficiaire [...] Mon budget est dans le vert ! À aucun moment on me demande en quoi consiste ma prise en charge, quelle est sa philosophie. À la limite, je peux leur tenir mon discours très radical sur l'émancipation du patient et la remise en cause du pouvoir médical, ils ne m'en tiendraient nulle rigueur, si éventuellement ils comprenaient ce propos, car je suis ce bon élève [...],

\footnotetext{
${ }^{24}$ Alors même que le docteur Millot estime que les hospitalisations sont la plupart du temps inutiles. Ce qui aboutit au paradoxe suivant, qu'il dénonce avec un ton ironique pour souligner l'absurdité de la situation : «On fait hospitaliser des gens pour pouvoir faire ce que l'on veut faire et l'on dit en même temps qu'il faut arrêter d'hospitaliser des gens ».

${ }^{25}$ Ce qui constitue, en soi, un paradoxe de taille puisque le professionnel de santé a presque tout pouvoir dans une situation qui se veut égalitaire.
} 
nous sommes rentables donc tout va bien [...]. La posture médicale, la relation de soin, ils s'en fichent !»

L'expression «bon élève » est assez significative de la stratégie de cet acteur, que l'on qualifie de stratégie du compliant insoumis. Celle-ci consiste à jouer le jeu du système pour mieux le contourner: "C'est dans la compliance au système que l'on peut trouver l'insoumission ». Ce qui signifie, au fond, que le «formalisme techniciste et administratif » de l'ETP (Barrier, 2014) offre paradoxalement un gain d'autonomie et d'innovation aux professionnels de santé qui veulent ou plutôt qui savent le contourner.

\section{De la reproduction indéfinie de l'ordre social}

Le dispositif du «médecin ignorant» comporte ainsi, comme on l'a souligné tout au long de notre propos, des lignes de fuite et paradoxes qui peuvent avoir des effets inverses à ceux qui sont initialement escomptés. La rupture avec la domination et l'ordre existant est loin d'être totale. La posture rancièrienne du médecin ignorant se trouve en effet confrontée à une « reproduction indéfinie de l'ordre social » qui se repère à différents niveaux (Nordmann, 2008) que je vais étudier.

\section{L'observance d'émancipation}

Certains patients viennent au Cétrabète pour la posture d'émancipation que propose l'équipe médicale. C'est le cas de Natasha, jeune salariée dans une entreprise :

«Je suis venue au Cétrabète parce que je savais qu'ils avaient des pratiques un peu particulières et je n'en pouvais plus des diététiciens qui disaient "arrêtez de manger ceci ou cela" et qui me culpabilisaient [...]. Je suis venue pour cela. J'avais besoin de mieux me connaître, de reprendre confiance en moi et de me réapproprier mon corps. »

Mais le cas de Natasha est rare. Le paradigme émancipateur ne vient pas spontanément des patients, mais est imposé par les professionnels de santé ${ }^{26}$. C'est ce que semble affirmer Michelle, l'une des patientes interrogées qui semble vouloir être guidée par le corps médical :

«On nous apprend à arrêter de dire "il faut". Mais au départ, ça n'est pas facile. Nous, ce que l'on veut, c'est perdre du poids et savoir ce qu'il faut faire pour cela. Et cette autre façon de voir les choses, ça nous tombe dessus et on ne le veut pas forcément au début [...]. On a tellement appris à faire des régimes, qu'on a presque envie de leur demander: "Vous ne pourriez pas nous donner des petites recettes ?" Ah, on est impossibles (rires).»

\footnotetext{
${ }^{26}$ De même, le professionnel tend parfois à renforcer la politisation des échanges. Non pas parce qu'il conflictualise les échanges, mais parce qu'il opère quelques montées en généralité (Hamidi, 2006) à partir des propos des patients : «On est dans une société qui veut toujours nous pousser à la consommation », «C'est une forme de racisme anti-gros », etc.
} 
Cette volonté d'imposition est reconnue par les professionnels de santé. Comme le souligne le docteur Millot lui-même au cours d'un entretien : "Il est vrai qu'il y a deux chapelles, et que l'on a tendance à vouloir imposer à nos patients notre façon de penser, mais c'est parce que l'on a raison! ». Ceci se perçoit parfois, dans les séances d'ETP, à travers certains échanges entre le professionnel de santé et ses patients. Il en va ainsi, par exemple, lorsqu'une patiente se plaint de l'attitude de son médecin traitant: "On vous regarde de haut, on vous toise, y compris les professionnels de santé. Vous savez, mon médecin m'a envoyée aux alcooliques anonymes, c'est vous dire ! » Le professionnel de santé réagit alors, divisant implicitement le corps professionnel en deux catégories, un sourire complice au coin des lèvres : "Oui, en effet [...]. Il y a des professionnels de santé qui fonctionnent comme ça [...]. Ça n'est pas notre façon de faire ».

Le paradoxe est ici de taille : les professionnels de santé qui s'inscrivent dans un paradigme émancipateur, selon lequel le médecin n'est pas celui qui dicte à son patient ce qu'il faut faire, imposent malgré tout à leurs patients ce paradigme duquel ils peuvent difficilement sortir. Paradigme selon lequel la perte de poids ou le suivi d'un régime sont moins importants que la prise de conscience des déterminismes sociaux qui pèsent sur le corps de l'individu.

Ce paradoxe se repère au détour de certains messages véhiculés par le diététicien lors des séances d'ETP : «Il faut vous faire confiance », «Pensez à vous », «Arrêtez de dire "il faut" ». Quand il prononce de telles phrases, le professionnel de santé demeure dans la position du sachant: il sait ce qu'il faut faire pour émanciper ses patients et agit en conséquence en leur dictant discrètement une marche à suivre. Même si ses messages s'inscrivent dans un cadre de pensée libertaire visant à rompre avec l'autolimitation de ses patients, le professionnel joue ici davantage le rôle de l'explicateur qui fait advenir à la surface ce que ses patients ignorent, que le rôle du maître ignorant. Il est un guide qui remet volontiers sa blouse blanche au vestiaire, mais demeure un guide. Ce qui constitue en soi une première entrave à la pensée rancièrienne qui veut que l'individu n'a nul besoin d'un maître pour s'émanciper. Pour Rancière, l'émancipation de l'individu ne peut en effet être guidée de l'extérieur, mais doit advenir des capacités de l'individu lui-même. Or, c'est le contraire qui se produit ici : certes, le professionnel de santé semble avoir confiance en la capacité de ses patients à s'émanciper eux-mêmes ${ }^{27}$, mais il continue dans le même temps à vouloir les émanciper. Au final, plutôt que de prescrire des traitements médicaux, il en vient presque et malgré lui à prescrire de l'émancipation. L'observance change donc d'objet et l'image du «bon patient» s'en trouve renversée. En effet, alors que le «bon patient» est traditionnellement celui qui, comme le démontre Aline Sarradon-Eck (2007), suit son traitement médical sans rechigner et se complaît dans une position de soumission à l'égard du discours médical traditionnel, il est ici celui qui parvient à s'émanciper à l'égard de ce discours médical traditionnel et à refuser sa servitude volontaire.

\section{Un dispositif verrouillé par le médical}

Si le médecin ignorant prescrit de l'émancipation, il impose aussi le cadre organisationnel qui la rend possible. En effet, quelle que soit la nature des interactions sociales qui se jouent dans cette ETP, la construction du programme et le déroulement des séances incombent uniquement à l'équipe médicale. Le postulat de l'égalité des savoirs se trouve donc, dans les

\footnotetext{
${ }^{27}$ Ce qui se repère à travers les questions ouvertes qu'il pose (pourquoi pensez-vous cela ? Qu'est-ce qui vous fait dire cela ?) ou certaines de ses formulations («C'est à vous de trouver le chemin », «C'est à vous de me dire ce que vous devez faire ») qui témoignent d'un travail de restructuration cognitive. Cette manière de procéder renvoie à des techniques de psychothérapie comportementale dont s'inspire l'équipe médicale du Cétrabète, en particulier la thérapie cognitivo-comportementale (TCC) ou la thérapie interpersonnelle (Wilfley et al., 2002).
} 
faits, confronté à une absence de démocratisation du dispositif qui reste dans les mains du pouvoir médical. Comme le souligne un patient : "Quand on arrive en ETP, on ne sait pas forcément ce que l'on va nous faire faire, c'est la surprise, la découverte ». Le processus décisionnel est ici décalé par rapport aux formes canoniques de la démocratie participative : il ne s'agit pas tant de participer à des décisions publiques (Cohen, Fung, 2004) sur le devenir d'un projet collectif, que de participer à des décisions concernant sa propre santé, son propre corps. Participer, c'est d'abord participer à la gestion de sa maladie. Pour le reste, les professionnels de santé décident de tout, y compris des patients qui sont autorisés ou non à assister aux séances collectives d'ETP.

Plusieurs critères entrent en ligne de compte, mais ces critères ne sont pas formalisés et sont laissés à la discrétion du corps médical. Or, mes entretiens et mes observations participantes offrent des indications sur les «modes » de sélection des participants. Comme le soulignent les salariés du Cétrabète, il s'agit majoritairement de "personnes qui ont déjà un rapport réflexif sur leur santé et leur corps », qui «n'ont pas de problème mental », ou encore «qui sont capables d'échanger et de discuter collectivement». Ce qui rejoint ici l'argument classique des premiers défenseurs de la démocratie délibérative selon lesquels la qualité de la délibération peut justifier la fermeture du cercle des délibérants. Dans les faits, ce dispositif émancipateur ne s'adresse donc pas à n'importe qui et un «filtrage » sur des critères à la fois psychologiques et sociaux s'opère de manière assez importante. C'est là une différence majeure avec les deux autres dispositifs d'ETP que j'ai pu observer dans le cadre de ma thèse, dont le seul critère de sélection des participants était d'être atteint d'une maladie chronique et d'avoir obtenu l'accord préalable de son médecin traitant. Alors que dans ces deux dispositifs d'ETP, que j'ai qualifiés de paternalistes, la très grande majorité des participants sont des hommes et femmes d'une cinquantaine d'années sans activité professionnelle ${ }^{28}$, la majorité des participants à l'ETP du Cétrabète sont des actifs entre 30 et 50 ans, dont certains ont un bagage culturel conséquent : on note, par exemple, la présence d'une professeure d'espagnol dans le supérieur et d'un bibliothécaire. Certains ont même une certaine familiarité avec l'univers médical : l'une des participantes est assistante sociale et l'autre est aide-soignante.

Cette sélection sociale se joue notamment lors de la consultation médicale qui constitue, de fait, une porte d'entrée dans certains dispositifs de soins participatifs qui n'existe pas dans les formes canoniques de la démocratie participative. Ce n'est généralement qu'au bout d'une dizaine de consultations médicales que les patients du Cétrabète les plus « réflexifs » sont invités à faire de l'ETP. La consultation médicale est donc un moment essentiel où s'élabore une relation de confiance entre le professionnel de santé et son patient qui détermine la suite du parcours du patient. Elle est l'antichambre d'un dispositif qui demeure cadenassé et descendant $^{29}$ : seul le professionnel de santé détient le secret des étapes que doit franchir son patient pour participer à l'ETP. Secret qu'il décide ou non de lui dévoiler s'il estime que ce dernier est apte à rejoindre le dispositif.

\section{La division sociale des intelligences}

Évidemment, cette sélection sociale est contradictoire avec la perspective rancièrienne d'émanciper les dominés. Dans cette ETP, on émancipe les émancipés et l'on renforce ainsi, malgré une exigence affichée d'égalité, le phénomène d'exclusion sociale déjà pointé du doigt

\footnotetext{
${ }^{28}$ Une majorité d'entre eux éprouvait quelques difficultés avec le maniement de la langue française.

${ }^{29}$ La consultation médicale constitue, de fait, une porte d'entrée dans les dispositifs participatifs de soins qui n'existe pas dans les outils canoniques de la démocratie participative.
} 
au sujet de la démocratie participative (Sander, 1997 ; Mouffe, 2000). Ce constat est partagé par le docteur Millot qui cultive à ce sujet un sentiment d'impuissance, voire d'échec :

\begin{abstract}
«On pense à tous ces gens devant lesquels je suis le plus démuni, qui ont 500 mots de vocabulaire. Des gens complètement exclus de toute représentativité, dans des situations économiques et sociales inextricables et devant lesquels on est devant un constat d'échec. Mais c'est à eux que l'on a envie de dire "Mais comment est-ce que l'on peut vous aider ?"! On parle d'émancipation, c'est notre objectif, mais ces gens sont peu capables de dire et de produire une pensée complexe! Sur ces gens, je suis complètement démuni [...] Ma posture marche bien avec ceux qui sont disposés à cela. »
\end{abstract}

On voit en effet se dessiner très nettement une division sociale entre le public des « sans », qui est celui qui résiste le plus souvent au discours émancipateur de l'équipe médicale et se complaît dans une forme de servitude volontaire, et le public des patients «dominants », qui est un public particulièrement disposé à entendre le discours émancipateur et à le cultiver. Cette reproduction d'une hiérarchisation sociale, contre laquelle voudrait lutter efficacement l'équipe médicale, met à mal l'exigence de l'égalité des intelligences qui se trouve ici réduite à n'avoir d'effet performatif qu' avec un public averti. Paradoxalement, le docteur qui prône la posture d'égalité intériorise dans le même temps la hiérarchisation sociale des intelligences et renforce ainsi le cercle des impuissances. Plutôt que la puissance d'un discours sur l'égalité, on fait ici face à la puissance d'un discours sur l'inégalité.

Il convient toutefois d'apporter une nuance : il peut évidemment arriver que des patients avec des capitaux culturels, économiques ou sociaux importants restent sourds au discours émancipateur du médecin ignorant. La position sociale de l'acteur ne détermine pas nécessairement, ou en tout cas pas totalement, sa capacité à accepter ce discours. La note ciaprès, issue de ma première rencontre avec le docteur Millot, en témoigne.

\title{
Encadré 4. Observation tirée d'une journée sur l'éducation thérapeutique du patient
}

Après un discours très humble du docteur Millot qui affirme que le professionnel de santé doit se mettre sur un pied d'égalité avec son patient et ne pas imposer de message médical ou d'impératifs à suivre, une dame de l'auditoire intervient avec un certain agacement dans la voix :

« Je suis insuffisante rénale depuis 32 ans, et j'ai fait des études de pharmacie, donc je sais de quoi je parle, mais je suis étonnée par votre discours, docteur. Jusqu'à présent j'adhérais totalement à ce que vous disiez parce que je pensais, au fond de moi : "Enfin un médecin qui se met un petit peu à notre portée et qui nous écoute". Mais là, ça va trop loin [...]. Le but de l'éducation thérapeutique, c'est d'apprendre au nouveau patient à avoir un minimum d'observance pour améliorer sa qualité de vie et la prolonger. Si vous voulez que les patients écoutent et améliorent leur façon de faire, il faut parfois imposer les choses! Imposer un savoir médical! Enfin, il faut arrêter les bêtises ! Il faut que les soignants soient là pour nous apprendre, nous dire ce qu'il faut faire, parce que nous, on n'a pas fait toutes ces études-là, nous les patients et malades. C'est votre responsabilité de professionnel ! [...]. La personne malade a besoin d'être rassurée, guidée. » 
La patiente, qui bénéficie visiblement d'un capital médical important, critique ici non pas le trop-plein de pouvoir médical, mais son effacement. Comment faut-il le comprendre ? À travers le discours de cette patiente, on se rend compte que le vécu de la maladie et son caractère anxiogène peuvent créer une forme de résistance consciente et assumée au discours émancipateur du médecin. Le rapport particulier que l'individu entretient avec son corps peut en effet constituer un facteur déterminant, en plus des facteurs économiques ou sociaux, au refus d'émancipation. Dans le cas spécifique de l'obésité, la volonté de certains patients de perdre du poids à tout prix peut les rendre sourds au discours émancipateur de l'équipe médicale. Comme le souligne une psychologue au cours d'une réunion : «Le culte de la minceur peut toucher tout le monde » (Le Breton, 2010), et le docteur Millot d'ajouter : «Il suffit qu'un chirurgien ait dit à un patient qu'il acceptait de l'opérer et qu'il fallait juste l'accord d'un nutritionniste, c'est-à-dire moi, pour que mon discours reste inaudible ». Dans ces conditions, et même si l'équipe médicale tente au maximum de percer à jour les raisons profondes qui motivent cette volonté, le corps médical se soumet à la volonté du patient ${ }^{30}$. L'une des raisons tient au fait, à nouveau, que l'activité médicale demeure une activité consultative qui doit rester lucrative : «Il ne faut pas non plus les faire fuir, il faut que ça tourne », reconnaît le diététicien. D'ailleurs, comme le souligne le docteur Millot :

«Les patients qui refusent de s'émanciper sont souvent ceux que l'on fait opérer, car on ne parvient à rien. Mais ils nous sont utiles, heureusement qu'ils sont là, parce que leurs opérations ou leurs hospitalisations rapportent du budget supplémentaire. $»^{31}$

Mais l'opposition binaire entre les patients qui acceptent l'émancipation et ceux qui la refusent est aussi une manière de simplifier la réalité. Dans les faits, le discours émancipateur du professionnel de santé n'est presque jamais totalement accepté ou définitivement acquis : certains patients, qui semblent pourtant se conformer à ce discours et à la posture du médecin ignorant en ETP, cherchent occasionnellement à replacer le médecin dans la posture de l'expert médical qui doit fournir des réponses médicales à la demande de perte de poids. Ainsi donc, tout se passe comme si, avec certaines catégories de patients, le médecin devait sans cesse se battre contre la tentation de ses patients de lui attribuer, malgré lui, son rôle de «sachant» et d'éducateur ${ }^{32}$. En ce sens, la posture du médecin ignorant constitue davantage un idéal régulateur qui guide la pratique médicale qu'une réalité constante et intangible.

\section{Une autre forme de pouvoir}

La posture du médecin ignorant fait par ailleurs face à ce qui constitue sans doute son plus grand paradoxe: celui de renforcer le pouvoir du médecin. En effet, même si les professionnels du Cétrabète s'efforcent de lutter contre le paternalisme médical et mettent en place des pratiques «émancipatrices », leur pouvoir ne cesse de se renforcer. Mais ce pouvoir

\footnotetext{
${ }^{30}$ Le contraire serait d'ailleurs paradoxal, puisque cela reviendrait à vouloir imposer à tout prix, contre la volonté du patient, un impératif d'émancipation.

${ }^{31}$ Ce qui rejoint ce que certaines études ont déjà démontré, à savoir que les impératifs de rentabilité engendrent une sélection à la fois des actes à réaliser et des patients à prendre en charge. Pour le dire autrement, les professionnels ont tendance à se centrer sur les patients les plus rémunérateurs (Ruggie, 1992).

${ }^{32} \mathrm{Ce}$ qui tient certainement au caractère prescriptif du pouvoir médical, qui le différencie en ce sens du pouvoir politique.
} 
prend une autre forme que celui du paternalisme traditionnel : il s'agit d'un leadership, d'un pouvoir charismatique que les professionnels de santé acquièrent à travers leur discours «novateur », «marginal », « humble ». C'est paradoxalement en refusant les attraits du pouvoir médical «traditionnel » que ces professionnels de santé conquièrent cet autre type de pouvoir. Ils acquièrent de nouvelles rétributions symboliques qui tendent à renforcer leur influence.

Ceci se perçoit dans le discours de certains patients, notamment chez ceux que l'on a qualifiés « d'inconditionnels », qui se montrent reconnaissants à l'égard de ces professionnels de santé et les font exister comme des «professionnels à part». Certains patients vouent même une certaine admiration, ainsi qu'une forte affection, à ces professionnels de santé qu'ils qualifient de «belles personnes », « de professionnels exceptionnels», et/ou de «sauveurs ». C'est le cas de Mylène, lorsque je l'interroge sur son expérience d'ETP :

«Ils n'ont rien à voir avec la diététicienne que je suis déjà allée consulter et qui m'a fait culpabiliser, comme la plupart des professionnels. C'est le jour et la nuit avec eux [...]. Je les admire, ce ne sont pas des professionnels comme les autres. Ce qu'ils font, c'est fou. Ce sont de belles personnes [...]. Je ne parviens pas à leur dire combien je les aime [...]. Ils m'ont apporté énormément dans ma vie [...] ils m’ont sauvée. »

L'admiration et l'affection de Mylène semblent être ici d'autant plus fortes qu'elles contrastent avec «l'aversion » qu'elle éprouve à l'égard d'autres professionnels de santé. Les patients sont donc des vecteurs importants de cette nouvelle forme du pouvoir professionnel, dans la mesure où ils tendent, encore et toujours, à sacraliser les professionnels de santé et leurs pratiques. Mais les professionnels cherchent également, consciemment ou inconsciemment, à renforcer leur leadership à travers la démonstration de leur «différence » avec les autres professionnels de santé. Le docteur Millot assume très clairement cette recherche de notoriété qui passe, paradoxalement, par l'affirmation de son humilité :

«Quelque part, à travers tout ça, je cherche une certaine notoriété. Je recherche à être entendu et reconnu, c'est certain [...]. C'est contradictoire, paradoxal. Mais oui, je recherche ça. Bien évidemment. »

Cette recherche de notoriété est à replacer dans un contexte plus général de déclin du « prestige social » de la profession de généraliste (Hassenteufel, 1997). Au fond, à travers la posture du médecin ignorant, l'un des objectifs est de redorer l'image ou la légitimité du pouvoir médical. Même s'il s'agit ici d'une autre forme de pouvoir médical (Tessier, 2012).

\section{Conclusion}

Au final, malgré le caractère ouvertement libertaire et l'égalitarisme ranciérien qui entourent ce dispositif de soin, celui-ci est condamné à rester encastré dans la reproduction de l'ordre médical existant. Le cadre pédagogique de la pensée rancièrienne résiste mal à la réalité de la pratique et de la responsabilité médicale. D'ailleurs, pouvait-il réellement y résister ? Nous 
pouvons en douter dans la mesure où tout le monde n'a pas le luxe de pouvoir «jouer » à l'ignorant tout en continuant de bénéficier, à l'instar des professionnels de santé, d'un certain prestige et d'une reconnaissance sociale. Le rôle du médecin ignorant est en soi inégalitaire puisqu'il est précisément l'apanage des professionnels de santé. Si l'on ajoute à cela le verrouillage du dispositif par le pouvoir médical, notre détour par ce dispositif buissonnier nous ramène aux conclusions d'un grand nombre de travaux critiques sur la démocratie participative qui mettent en lumière l'écart entre les idéaux normatifs qui peuvent justifier la mise en place des dispositifs participatifs, notamment en termes de réduction des inégalités (Boulding, Wampler, 2010), et la réalité effective de leur fonctionnement (Nonjon, 2006 ; Blatrix, 2009). Ce qui ne remet cependant pas en cause la portée heuristique de l'analyse que l'on propose dans cet article.

Cette analyse permet d'abord de peaufiner la réflexion sur le caractère buissonnier de certaines pratiques participatives. Il démontre notamment, à rebours d'une conception « intuitive » de la participation buissonnière, que le caractère buissonnier de la participation n'est pas inversement proportionné à son degré d'institutionnalisation. Il s'agit donc de sortir d'une vision binaire qui opposerait, d'un côté, une participation institutionnalisée à une participation non reconnue, clandestine.

Ce détour permet ensuite de mettre en relief des conditions particulières d'émergence d'un espace de parole favorable aux prises de conscience et à la baisse de la culpabilité des patients dans l'univers hospitalier: au-delà de facteurs endogènes à la pratique et à la relation médicales, comme la posture d'humilité du professionnel de santé ou encore le type de maladie traitée, ce sont aussi des facteurs exogènes à la relation médicale, comme la recherche de rentabilité et le profit économique réalisé par le service hospitalier, qui rendent possible et pérenne ce dispositif.

Ce détour permet également de mettre en lumière les particularités des dispositifs médicaux par rapport aux outils canoniques de la démocratie participative. Parmi ces particularités, on note l'existence de la consultation médicale comme passage obligé dans l'arène participative, la portée essentiellement individualisante du processus décisionnel qui s'attache à la santé de l'individu et non à une décision collective et/ou publique, le rôle central joué par le corps médical et certains enjeux professionnels dans le design du dispositif, les thématiques abordées et la teneur des échanges.

Enfin, ce détour permet de mettre en lumière certains impensés des travaux sur la démocratie participative qui, à quelques exceptions près (de Nanteuil, 2014), ne s'intéressent que très peu à l'expérience vécue, sensible et incorporée des individus, au pouvoir de l'intimité ou encore au caractère anxiogène de certaines arènes participatives. Alors que la littérature sur la participation, mis à part quelques travaux récents (Jacquet, 2015), fait très peu état des cas de rejet de l'impératif participatif par le public, si ce n'est pour supposer que cette "nonparticipation » se comprend d'abord et avant tout par des logiques d'exclusion sociale qui dépassent largement les dispositifs participatifs, notre analyse nous invite au contraire à déconstruire la désirabilité de la participation et de l'empowerment et à considérer différentes postures face au discours émancipateur. Dans le cas précis des dispositifs de soins, ce rejet peut se comprendre, on l'a vu, par la dépendance médicale occasionnée par la maladie chronique, par l'expérience vécue et sensible de cette maladie, mais aussi par les attendus sociaux concernant le pouvoir médical qui est d'abord et avant tout un pouvoir prescriptif. Il y a encore aujourd'hui, et ce malgré le passage d'une société moderne, caractérisée par la croyance dans la toute-puissance de la science, à une société dite «post-moderne » (Beck, 2001), caractérisée quant à elle par la prise de conscience des limites de la science et de ses 
effets indésirables, une véritable remise de soi au corps médical qui perdure. Comme le rappellent Clarisse Gervais, Mario Sanchez et William Lowenstein (2010) à ce sujet, « lorsque l'on s'en remet à un psychologue, plus encore à un médecin, on lui suppose un savoir, on attend de lui l'interprétation de signes disparates qui, mis bout à bout, poseront un diagnostic ». Cette spécificité du pouvoir médical, déjà bien indiquée dans les travaux de sociologie de la santé, nous rappelle un peu plus l'intérêt d'un décentrage du regard sociologique vers d'autres outils que les outils de la démocratie participative classique.

\section{Bibliographie}

Adam P., Herzlich C., 2012, Sociologie de la maladie et de la médecine, Paris, Armand Colin.

Azria E., 2013, « Le soignant et la standardisation des pratiques médicales », Laennec, 61 (3), p. 32-41.

Bacqué M.-H., Biewener C., 2013, L'empowerment, une pratique émancipatrice, Paris, La Découverte.

Barbot J., 2002, Les malades en mouvement. La médecine et la science à l'épreuve du sida, Paris, Balland.

Barrett R., 1998, La traite des fous, la construction sociale de la schizophrénie, Le PlessisRobinson, Institut Synthélabo pour le progrès de la connaissance.

Barrier P., 2014, Le patient autonome, Paris, Presses universitaires de France.

Beck U., 2001, La société du risque. Sur la voie d'une autre modernité, Paris, Aubier.

Bergeron H., Castel P. (dir.), 2010, Regards croisés sur l'obésité, Paris, Éditions de Santé/Presses de Sciences Po, p. 73-76.

Blatrix C., 2009, «La démocratie participative en représentation », Sociétés contemporaines, 74 (2), p. 97-119.

Boltanski L., Chiapello E., 1999, Le nouvel esprit du capitalisme, Paris, Gallimard.

Boulding C., Wampler B., 2010, «Voice, Votes, and Resources : Evaluating the Effect of Participatory Democracy on Well-being », World Development, 38 (1), p. 125-135.

Buissonnet-Verger G., 1997, L'éducation et la santé : essai de clarification conceptuelle, Montréal, S.P.I.D. Verger Inc., https://spid.com/acrobat/def_sante.pdf (accès le 30/01/2018).

Bury J. A., 1988, Éducation pour la santé. Concepts, enjeux, planifications, Bruxelles, De Boeck Université.

Canguilhem G., 1994, «Le statut épistémologique de la médecine », in G. Canguilhem, Études d'histoire et de philosophie des sciences, Paris, Vrin, p. 413-428.

Cerletti A., 2005, «La politique du maître ignorant : la leçon de Rancière », Le Télémaque, 27 (1), p. 81-88. 
Cohen J., Fung A., 2004, «Radical democracy», Swiss Political Science Review, 10 (3), p. 23-34.

Elger B., 2010, Le paternalisme médical : mythe ou réalité ? Aspects philosophiques et empiriques d'un phénomène persistant, Genève, Médecine \& hygiène.

Fassin D., Memmi D. (dir.), 2004, Le gouvernement des corps, Paris, Éditions de l'EHESS.

Fauquette A., 2016, Contribution à une analyse sociologique de l'effet sectoriel sur la participation: le cas du secteur sanitaire (2010-2016), thèse pour le doctorat de science politique, Université Lille 2.

Fischler C., 1990, L’homnivore, Paris, Odile Jacob.

Flora L., 2013, «Savoirs expérientiels des malades, pratiques collaboratives avec les professionnels de santé : état des lieux », Éducation permanente, 195, p. 59-72.

Foucault M., 1976, Histoire de la sexualité 1. La volonté de savoir, Paris, Gallimard.

Foucault M., 1994, «L'éthique du souci de soi comme pratique de la liberté », in D. Defert, F. Ewald (dir.), Foucault. Dits et écrits, vol. IV, Paris, Gallimard, p. 708-729.

Freire P., 1974, Pédagogie des opprimés. Suivi de Conscientisation et Révolution, Paris, Maspero.

French J., 1990, «Boundaries and Horizons, the role of health education within health promotion », Health Education Journal, 49 (1), p. 7-1.

Gaxie D., 1996, «Gouvernabilité et transformations structurelles des démocraties », in CURAPP, La Gouvernabilité, Paris, Presses universitaires de France, p. 249-273.

Genard J.-L., Cantelli F., 2008, «Êtres capables et compétents : lecture anthropologique et pistes pragmatiques», SociologieS, Théories et recherches, 27 avril 2008, http://journals.openedition.org/sociologies/1943 (accès le 30/01/2018).

Gervais C., Sanchez M., Lowenstein W., 2010, «Addictions et politis », in H. Bergeron, P. Castel (dir.), Regards croisés sur l'obésité, Paris, Éditions de Santé/Presses de Sciences Po, p. 73-76.

Goffman E., 1979, Asiles. Études sur la condition sociale des malades mentaux et autres reclus, trad. L. et C. Lainé, Paris, Éditions de Minuit.

Green W. H., Simons-Morton B. G., 1984, Introduction to Health education and Health Promotion, New York, Macmillan Publishing Company.

Grimaldi A., Caillé Y., Pierru F., Tabuteau D., Assal J.-Ph., 2017, Les maladies chroniques. Vers la troisième médecine, Paris, Odile Jacob.

Hamidi C., 2006, «Éléments pour une approche interactionniste de la politisation. Engagement associatif et rapport au politique dans des associations locales issues de l'immigration », Revue française de science politique, 56 (1), p. 5-25.

Hassenteufel P., 1997, Les médecins face à l'État. Une comparaison européenne, Paris, Presses de Sciences Po. 
Huyard C., 2012, Rare. Sur la cause politique des maladies peu fréquentes, Paris, Éditions de l'EHESS.

Illich I., 1975, Némésis médicale, Paris, Seuil.

Jacquet V., 2017, «Explaining non-participation in deliberative mini-publics », European Journal of Political Research, 56 (3), p. 640-659.

Jouve B., 2005, «La démocratie en métropoles : gouvernance, participation et citoyenneté », Revue française de science politique, 55 (2), p. 317-337.

Klein A., 2014, «Au-delà du masque de l'expert. Réflexions sur les ambitions, enjeux et limites de l'Éducation Thérapeutique du Patient», Rééducation orthophonique, 259, https://hal.archives-ouvertes.fr/hal-01228239

Larchet N., 2015, «De l'urgence sociale à l'utopie sanitaire. La construction sociale de l'obésité et l'occultation de la faim dans les villes américaines, Knoxville, 1981-1985 », Actes de la recherche en sciences sociales, 208, p. 40-61.

Le Breton D., 2010, «L'obésité entre dissidence et résistance », in H. Bergeron, P. Castel (dir.), Regards croisés sur l'obésité, Paris, Éditions de santé/Presses de Sciences Po, p. 19-26.

Lefève C., 2015, «Georges Canguilhem : se projeter en situation de malade », in Lefève C., Benaroyo L., Worms F., Les classiques du soin, Paris, Presses universitaires de France, p. 228.

Lorig K. R., Lubeck D., Kraines R. G., Seleznick M., Holman H. R., 1985, «Outcomes of self-help education for patients with arthritis », Arthritis and Rheumatism, 28 (6), p. 680-685.

Lorig K. R., Sobel D. S., Laurent D., Hobbs M., 2001, «Effect of a self-management program on patients with chronic disease », Effective Clinical Practice, 4, p. 256-262.

Lorig K. R., Sobel D. S., Stewart A. L., 1999, «Evidence suggesting that a chronic disease self-management program can improve health status while reducing hospitalization », Medical Care, 37, p. 5-14.

Mas B., Pierru F., Smolski N., 2011, L’hôpital en réanimation. Le sacrifice organisé d'un service public emblématique, Paris, Éditions du Croquant.

Miller L. V., Goldstein G., 1972, «More efficient care of diabetic patients in a countryhospital setting », New England Journal of Medicine, 286 (26), p. 1388-1394.

Mouffe C., 1999, «Deliberative Democracy or Agonistic Pluralism », Political Sciences Series, 72, p. 1-17.

Nanteuil (de) M., 2014, «Ce corps qui manque à la représentation. Entre démocratie participative et critique artistique, les nouvelles scènes de l'expérience politique », Participations, 9, p. 177-205.

Nonjon M., 2006, Quand la démocratie se professionnalise : enquête sur les experts de la participation, thèse pour le doctorat de science politique, Université Lille 2.

Nordmann C., 2008 [2006], Bourdieu/Rancière, la politique entre sociologie et philosophie, Paris, Éditions Amsterdam.

Poulain J.-P., 2009, Sociologie de l'obésité, Paris, Presses universitaires de France. 
Rancière J., 2004 [1987], Le maître ignorant. Cinq leçons sur l'émancipation intellectuelle, Paris, 10-18.

Régnier F., 2010, «Obésité et clivages sociaux: l'approche sociologique », in H. Bergeron, P. Castel (dir.), Regards croisés sur l'obésité, Paris, Éditions de Santé/Presses de Sciences Po, p. 41-52.

Ricœur P., 1990, Soi-même comme un autre, Paris, Seuil.

Ricœur P., 2004, Parcours de la reconnaissance, Paris, Stock.

Rogers C., 1952, «Client-Centered Psychotherapy », Scientific American, 187, p. 1-7.

Ruggie M., 1992, «The Paradox of Liberal Intervention : Health Policy and the American Welfare State », American Journal of Sociology, 97 (4), p. 919-944.

Saillant F., Gagnon E., 1996, «Le self-care : de l'autonomie-libération à la gestion du soi : le soin comme objet problématique », Sciences sociales et santé, 14 (3), p. 17-46.

Sanders L., 1997, « Against Deliberation », Political Theory, 25 (3), p. 347-376.

Sarradon-Eck A., 2007, «Le sens de l'observance. Ethnographie des pratiques médicamenteuses de personnes hypertendues », Sciences Sociales et Santé, 25 (2), p. 5-36.

Schumaker C. J. Jr., 1977, «The health services system : Dilemmas and potential solutions », Social Thought, 3 (3), p. 19-30.

Strauss A., 1992, La trame de la négociation. Sociologie qualitative et interactionniste, Paris, L'Harmattan.

Tessier S., 2012, Les éducations en santé. Éducation en santé, éducation thérapeutique, éducation à porter soins et secours, Paris, Maloine.

Tourette-Turgis C., 2015, L'éducation thérapeutique du patient. La maladie comme condition d'apprentissage, Louvain-la-Neuve, De Boeck supérieur.

Van Ballekom K., 2008, L'éducation du patient en hôpital. L'autonomie du patient : de l'information à l'éducation, Godine, Centre d'Éducation du Patient asbl, http://ipcem.org/img/ouvrages/vanballekom.pdf (accès le 30/01/2018).

Wilfley D. E., Welch R. R., Stein R. I., Spurrell E. B., Cohen L. R., Saelens B. E., Dounchis J. Z., Frank M. A., Wiseman C. V., Matt G. E., 2002, «A Randomized Comparison of Group Cognitive-Behavorial Therapy and Group Interpersonal Psychotherapy for the Treatment of Overweight Individuals with Binge-Eating Disorder », Archives of general psychiatry, 59 (8), p. 713-721.

Zola I.-K., 1972, « Medicine as an Institution of Social Control », Sociological Review, 20 (4) p. 487-504.

\section{Abstract - Rancière Applied to Healthcare : Expertise, Ignorance and Emancipation in a Participative Care Method}


This article examines a therapeutic patient education method designed and created by a libertarian doctor influenced by Jacques Rancière's "ignorant schoolmaster" model, with the aim being to radically break with the traditional codes of the medical relationship and eliminate any form of imposition of biopower. More specifically, the article explores the ambiguities of this alternative approach which, while it does tend to reduce the asymmetry of the medical relationship, only emancipates those who are already emancipated (autonomous), and fails to entirely break with traditional forms of doctor-patient domination. The reality of medical practice and doctors' responsibility toward their patients stand in the way of Rancière's egalitarianism and require constant compromise with the hospital establishment. The leeway left by the hospital establishment also simultaneously accentuates the transgressive nature of this method.

\section{Keywords}

Expertise, Ignorance, Emancipation, Therapeutic Education

Alexandre Fauquette est docteur en science politique rattaché au Centre d'Études et de Recherches Administratives, Politiques et Sociales (Université de Lille, CNRS, UMR 8026, CERAPS) et postdoctorant en sociologie à la Maison Européenne des Sciences de l'Homme et de la Société Lille-Nord de France (MESHS), sous contrat avec le CNRS. Sa thèse questionne les particularismes de la participation dans le domaine de la santé, et sa recherche postdoctorale porte sur un projet de réhabilitation urbaine autour des questions alimentaires. Il a notamment publié un article sur la régionalisation sanitaire au prisme des concurrences institutionnelles.

\section{Mots clés}

Expertise, ignorance, émancipation, éducation thérapeutique. 